International Journal of Modern Physics A

(C) World Scientific Publishing Company

\title{
A Two Higgs Bi-doublet Left-Right Model With Spontaneous $C P$ Violation
}

\author{
Yue-Liang Wu \\ Kavli Institute for Theoretical Physics China, Institute of Theoretical Physics, \\ Chinese Academy of Sciences, Beijing 100080, P.R.China \\ Yu-Feng Zhou \\ Korea Institute for Advanced Study, Seoul, 130-722, Korea
}

\begin{abstract}
We discuss a left-right symmetric model with two Higgs bi-doublet and spontaneous $\mathrm{P}$ and $C P$ violation. The flavor changing neutral currents is suppressed by assuming approximate global $U(1)$ family symmetry. We calculate the constraints from neural $K$ meson mass difference $\Delta m_{K}$ and demonstrate that a right-handed gauge boson $W_{2}$ contribution in box-diagrams with mass around $600 \mathrm{GeV}$ is allowed due to a negative interference with a light charged Higgs boson around $150 \sim 300 \mathrm{GeV}$. The $W_{2}$ contribution to $\epsilon_{K}$ is suppressed from appropriate choice of additional $C P$ phases appearing in the right-handed Cabbibo-Kobayashi-Maskawa(CKM) matrix. The model is found fully consistent with $B^{0}$ mass difference and the mixing-induced $C P$ asymmetry measurements.
\end{abstract}

PACS numbers: $12.60 . \mathrm{Fr} ; 13.25 . \mathrm{Hw} ; 11.30 . \mathrm{Hv}$;

Since the discovery of parity violation fifty years agd 1 , the study of symmetry and symmetry breaking has played a central role in particle physics. With the hypothesis that parity is a good symmetry at high energy scale, a minimal left-right symmetric model was proposed based on the gauge group $S U(2)_{L} \times$ $S U(2)_{R} \times U(1)_{B-L}{ }^{2}$ with one Higgs bi-doublet. In such a model, parity violation is implemented through spontaneous symmetry breaking of the right-handed gauge sector. It is natural to require that in the left-right model, $C P$ violation is also a consequence of spontaneous symmetry breaking. However, a spontaneous $C P$ violating left-right model with only one Higgs bi-doublet is severely constrained by low energy phenomenology as it generates too much flavor changing neutral current(FCNC). For instance, the neutral kaon mass difference $\Delta m_{K}$ requires that the right-handed gauge bosons must be very heavy above $2 \mathrm{TeV}$ and the lightest neutral Higgs boson must be above $10 \mathrm{TeV}$ to suppress FCNC; the predicted CKM matrix elements do not coincide with the B factory measurements; and the the condition for the spontaneous $C P$ violation requires an unnatural fine tuning of the Higgs potential.

Motivated by the general two-Higgs-doublet model(2HDM) as a model for spon- 
taneous $C P$ violation, we extend the one Higgs bi-doublet left-right model to a two Higgs bi-doublet model with spontaneous $C P$ violation $\underline{6}$. The two Higgs bi-doublet fields are defined as

$$
\phi=\left(\begin{array}{cc}
\phi_{1}^{0} & \phi_{1}^{+} \\
\phi_{2}^{-} & \phi_{2}^{0}
\end{array}\right), \quad \chi=\left(\begin{array}{cc}
\chi_{1}^{0} & \chi_{1}^{+} \\
\chi_{2}^{-} & \chi_{2}^{0}
\end{array}\right) \quad:(2,2,0) .
$$

with most general Yukawa interaction for quarks

$$
\mathcal{L}_{Y}=-\sum_{i, j} \bar{Q}_{i L}\left(\left(y_{q}\right)_{i j} \phi+\left(\tilde{y}_{q}\right)_{i j} \tilde{\phi}+\left(h_{q}\right)_{i j} \chi+\left(\tilde{h}_{q}\right)_{i j} \tilde{\chi}\right) Q_{j R},
$$

where $\tilde{\phi}(\tilde{\chi})=\tau_{2} \phi^{*}\left(\chi^{*}\right) \tau_{2}$. Parity and $C P$ symmetry require that all the Yukawa matrices are real and symmetric. We also adopt the popular choice of introducing two Higgs triplets $\left(\Delta_{L} \sim(3,1,2), \Delta_{R} \sim(1,3,2)\right)$ to break $S U(2)_{L} \otimes S U(2)_{R} \otimes$ $U(1)_{B-L}$ down to $U(1)_{e m}{ }^{2}$

To suppress FCNC, we shall follow the similar treatment in the general $2 \mathrm{HDM}^{4}$ by considering the mechanism of approximate global $U(1)$ family symmetry 5

$$
\left(u_{i}, d_{i}\right) \rightarrow e^{-i \theta_{i}}\left(u_{i}, d_{i}\right),
$$

which is motivated by the approximate unity of the CKM matrix. As an consequence, $y, \tilde{y}, h$ and $\tilde{h}$ are nearly diagonal matrices. We argue that after the spontaneous symmetry breaking, the two Higgs bi-doublet fields can have complex vacuum expectation values(VEVs) which leads to the following mass matrix for up-quarks

$$
M_{u}=y_{q} v_{1} e^{i \delta_{1}}+\tilde{y}_{q} v_{2} e^{-i \delta_{2}}+h_{q} w_{1} e^{i \varphi_{1}}+\tilde{h}_{q} w_{2} e^{-i \varphi_{2}},
$$

where $v_{1,2}$ and $w_{1,2}$ are VEVs for $\phi$ and $\chi$. The down-quark mass matrix is obtained by replacing $1 \leftrightarrow 2$. It follows that the resultant quark mixing matrices for lefthanded and right-handed quarks are of pseudo-manifest type before rephasing. After rephasing the left-handed CKM matrix $V^{L}$ to the standard form with only one $C P$ phase, there are five $C P$ phases $\alpha_{i},(i=1,2,3)$ and $\beta_{i},(i=1,2)$ appearing in the right-handed CKM matrix $V^{R}$

$$
V^{R}=\eta^{u}\left(\begin{array}{lll}
\left(V_{u d}^{L}\right)^{*} e^{2 i \alpha_{1}} & \left(V_{u s}^{L}\right)^{*} e^{i\left(\alpha_{1}+\alpha_{2}+\beta_{1}\right)} & \left(V_{u b}^{L}\right)^{*} e^{i\left(\alpha_{1}+\alpha_{3}+\beta_{1}+\beta_{2}\right)} \\
\left(V_{c d}^{L}\right)^{*} e^{i\left(\alpha_{1}+\alpha_{2}-\beta_{1}\right)} & \left(V_{c s}^{L}\right)^{*} e^{2 i \alpha_{2}} & \left(V_{c b}^{L}\right)^{*} e^{i\left(\alpha_{2}+\alpha_{3}+\beta_{2}\right)} \\
\left(V_{t d}^{L}\right)^{*} e^{i\left(\alpha_{1}+\alpha_{3}-\beta_{1}-\beta_{2}\right)} & \left(V_{t s}^{L}\right)^{*} e^{i\left(\alpha_{2}+\alpha_{3}-\beta_{2}\right)} & \left(V_{t b}^{L}\right)^{*} e^{2 i \alpha_{3}}
\end{array}\right) \eta^{d},
$$

where $\eta^{u, d}$ are quark mass sign matrices.

With enlarged Higgs sector, in this model there are two doubly charged Higgs $H_{i}^{++},(i=1,2)$, four singly charged Higgs particles $H_{i}^{+},(i=1, \ldots 4)$, six neutral scalars $h_{i}^{0},(i=1, \ldots 6)$ and four neural pseudo-scalars $A_{i}^{0},(i=1, \ldots 4)$. The singly charged and neutral scalars may have significant effects on mixings and $C P$ violation in quark sector. For simplicity, we shall work in a simple scenario that only one charged Higgs (labeled as $H^{+}$) is light enough to actively contribute to the box-diagrams in neutral meson mixing. Of particular, when the VEVs satisfy the conditions $v_{2} \ll v_{1}$ and $w_{2} \ll w_{1}$, many features of this model are similar to the general $2 \mathrm{HDM}$ with spontaneous $C P$ violation 4 . Therefore, we consider here the 
2HDM-like charged Higgs to be the lightest one, the corresponding Yukawa interaction is parametrized as follows

$$
\mathcal{L}_{C}=-\left(2 \sqrt{2} G_{F}\right)^{1 / 2} \bar{u}^{i}\left(\sqrt{m_{i}^{u} m_{k}^{u}} \xi_{i k}^{u} V_{k j}^{L} P_{L}-V_{i k}^{L} \sqrt{m_{k}^{d} m_{j}^{d}} \xi_{k j}^{d} P_{R}\right) d^{j} H^{+}+\text {H.c. }
$$

The small off-diagonal terms characterized in $\sqrt{m_{i}^{q} m_{j}^{q}} \xi_{i j}^{q}$ describe the breaking of the global $U(1)$ family symmetry 7 . We denote the diagonal elements as $\xi_{c} \equiv \xi_{22}^{u}$ and $\xi_{t} \equiv \xi_{33}^{u}$.

Let us discuss the low energy phenomenology. In $K^{0}$ mixing, the smallness of $\epsilon_{K}=2.28 \times 10^{-3}$ requires that the dominant $W_{1} W_{2}$ box-diagrams with internal $(c, c)$ quark must be nearly real. This can be satisfied by imposing

$$
\alpha_{1}-\alpha_{2}-\beta_{1} \simeq 0 \text {. }
$$

Since the $H^{+}$contribution to $(c, c)$ quark loop is always real, and the induced effective operator have different chirality, the $H^{+}$loop interferes always destructively with the $W_{1} W_{2}$ loop in the $C P$ conserving case of Eq. (5). This provides a possibility of a cancellation, which may greatly reduce the mass lower bounds for both $W_{2}$ and charged Higgs $H^{+}$. Taking only the dominant $H^{ \pm} H^{ \pm}$loop contribution into account, we find that a complete cancellation requires

$$
\eta_{c c}^{H} x_{c}\left|\xi_{c}\right|^{4} \frac{M_{2}^{2}}{m_{H}^{2}} \simeq-24\left[\eta_{1}^{L R}\left(\ln x_{c}+1\right)+\frac{\eta_{2}^{L R}}{4} \ln \frac{m_{W}^{2}}{M_{2}^{2}}\right] \frac{m_{K}^{2}}{\left(m_{s}+m_{d}\right)^{2}} \frac{B_{K}^{S}}{B_{K}} .
$$

A numerical calculation including all the contributions is shown in Fig.1a. Numerically, for $m_{H^{+}} \sim 150 \mathrm{GeV}$ and Yukawa coupling $\xi \sim 25$, the charged Higgs can compensate a opposite contribution from a light $W_{2}$ at $M_{2} \sim 600 \mathrm{GeV}$. The large Higgs contribution relies on the fact that the $H^{+} H^{+}$loop is proportional to $\left|\xi_{c}\right|^{4}$ which grows rapidly with $\left|\xi_{c}\right|$ increasing.
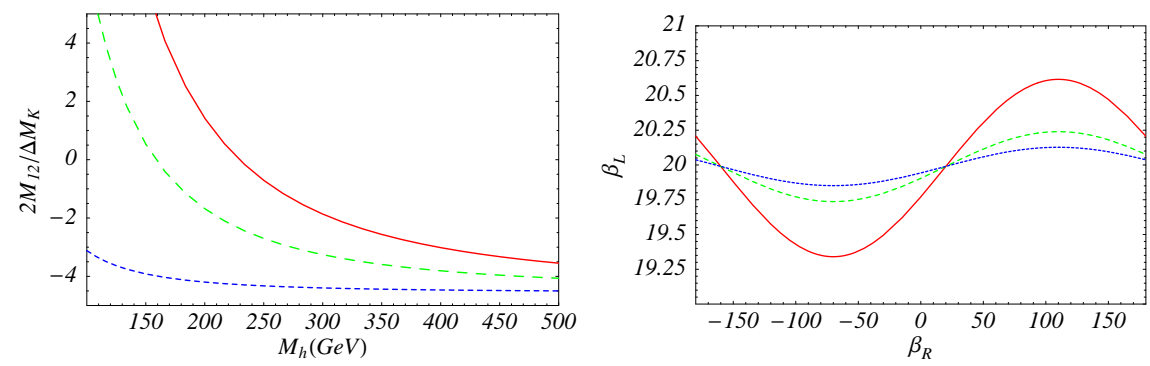

Fig. 1. (a)Left, sum of all loop contributions, including the SM contribution to the $2 M_{12}$ normalized to the measured $\Delta m_{K}$ with $M_{2}=600 \mathrm{GeV}$. Three curves corresponds to $\left|\xi_{c}\right|=30$ (solid), 25 (dashed) and 15 (dotted ) respectively. (b)Right, Values of $\beta_{L}$ as a function of $\beta_{R}$ for different $M_{2}$. Three curves correspond to $M_{2}=500 \mathrm{GeV}$ (solid), $1000 \mathrm{GeV}$ (dashed) and $1500 \mathrm{GeV}$ (dotted) respectively.

The situation is quite different for $B$ meson system. The $B^{0}$ meson mixing is dominated by internal $(t, t)$ quark loop. Due to the very weak mass-dependence of 
loop functions, the $W_{1} W_{2}$ loop are only a few percent of $W_{1} W_{1}$ loop in the SM at $m_{b}$ scale, which greatly suppresses its phenomenological significance in $B^{0}$ mixing. The charged Higgs contribution can be suppressed for small $\left|\xi_{t}\right|$. Within the Wolfenstein parametrization, we define $\beta_{L} \equiv \arg \left(V_{t d}^{L *} V_{t b}^{L}\right)$ which is to a high precision, one of the angles of the unitarity triangle. In the right-hand sector one can define a similar quantity $\beta_{R} \equiv \arg \left(V_{t d}^{R *} V_{t b}^{R}\right)$. With the pollution from $W_{1} W_{2}$ loop, the timedependent decay $B \rightarrow J / \psi K_{S}$ will only measures an effective phase angle $\sin 2 \beta_{\text {eff }}$. Using the measured experimental value of $\Delta m_{B}$ and $\beta_{\text {eff }}$ one can obtain the value of $\beta_{L}$ as a function of $\beta_{R}$ only. In fig.1b, we plot the $\beta_{L}$ as a function of $\beta_{R}$ with different values of $M_{2}$. One sees that for a light $W_{2}$ around $600 \mathrm{GeV}$, and $\beta_{R}$ varying from $-180^{\circ}$ to $180^{\circ}$, the modification to $\beta_{L}$ is less than $2^{\circ}$. Similarly, the change to the $\left|V_{t d}^{L}\right|$, which is calculated from $B^{0}$ mass difference is also very small. Thus this model can accommodate both the data of $\Delta m_{B}$ and $\sin 2 \beta_{J / \psi}$ with a light $W_{2}$, which is not possible to the left-right model with only one Higgs bi-doublet.

To summarize, we have investigated a general left-right symmetric model with two Higgs bi-doublets. This simple extension evades the stringent constraints from $K$ and $B$ meson mixing, and lowers the allowed mass of right-handed gauge boson closing to the current direct experimental search bound. Compared with the general $2 \mathrm{HDM}$, this model has even more sources of $C P$ violation in gauge sector, which may have interesting implications in lower energy phenomenology such as $\epsilon^{\prime} / \epsilon^{4}$ and puzzles in charmless $B$ decays 8 . The predicted new physics particles can be directly searched in upcoming LHC and future ILC experiments.

\section{References}

1. T.D. Lee and C.N. Yang, Phys. Rev. 104254 (1956). C.S. Wu, E. Ambler, R.W. Hayward, D. Hoppes and R.P. Hudson, Phys. Rev. 105, 1413 (1957).

2. J.C. Pati and A. Salam, Phys. Rev. D10 (1974) 275. R.N. Mohapatra and J.C. Pati, Phys. Rev. D11 (1975) 566. G. Senjanovic and R.N. Mohapatra, Phys. Rev. D12 (1975) 1502.

3. J. M. Frere, J. Galand, A. Le Yaouanc, L. Oliver, O. Pene and J. C. Raynal, Phys. Rev. D 46, 337 (1992). G. Barenboim, J. Bernabeu and M. Raidal, Nucl. Phys. B478, 527 (1996). P. Ball, J. M. Frere and J. Matias, Nucl. Phys. B572, 3 (2000).

4. Y.L. Wu and L. Wolfenstein, Phys. Rev. Lett. 731762 (1994). L. Wolfenstein and Y.L. Wu, Phys. Rev. Lett., 73, 2809 (1994). Y.L. Wu, Carnegie-Mellon Univ. report, CMUHEP94-01, hep-ph/9404241, 1994; Invited talk at 5th Conference on the Intersections of Particle and Nuclear Physics, St. Petersburg, FL, 31 May- 6 Jun 1994, published in Proceedings, pp338, edited by S.J. Seestrom (AIP, New York, 1995), hep-ph/9406306. Y. L. Wu and Y. F. Zhou, Phys. Rev. D 61, 096001 (2000).

5. L.J. Hall and S. Weinberg, Phys. Rev. D48, R979 (1993).

6. Y. L. Wu and Y. F. Zhou, arXiv:0709.0042 [hep-ph].

7. T.P. Cheng and M. Sher, Phys. Rev. D 35,3484(1987).

8. A. J. Buras, R. Fleischer, S. Recksiegel and F. Schwab, Phys. Rev. Lett. 92 (2004) 101804, Nucl. Phys. B 697, 133 (2004). Y. L. Wu and Y. F. Zhou, Phys. Rev. D 71, 021701 (2005). Phys. Rev. D 72, 034037 (2005) Y. L. Wu, Y. F. Zhou and C. Zhuang, Phys. Rev. D 74, 094007 (2006). arXiv:hep-ph/0606035 